\title{
Parallel-Tempering Monte-Carlo Simulation with Feedback-Optimized Algorithm Applied to a Coil-to-Globule Transition of a Lattice Homopolymer
}

\author{
K. Lewandowski, P. Knychała, M. Banaszak* \\ Faculty of Physics, A. Mickiewicz University \\ ul. Umultowska 85, 61-614 Poznan, Poland \\ *e-mail:mbanasz@amu.edu.pl \\ http://www.simgroup.amu.edu.pl
}

(Received: 15 March 2010; revised: 21 April 2010; accepted: 23 April 2010; published online: 4 May 2010)

\begin{abstract}
We present a study of the parallel tempering (replica exchange) Monte Carlo method, with special focus on the feedbackoptimized parallel tempering algorithm, used for generating an optimal set of simulation temperatures. This method is applied to a lattice simulation of a homopolymer chain undergoing a coil-to-globule transition upon cooling. We select the optimal number of replicas for different chain lengths, $N=25,50$ and 75, using replica's round-trip time in temperature space, in order to determine energy, specific heat, and squared end-to-end distance of the homopolymer chain for the selected temperatures. We also evaluate relative merits of this optimization method.
\end{abstract}

Key words: Monte Carlo, parallel tempering, replica exchange, feedback-optimized, polymer, single chain

\section{INTRODUCTION}

Monte Carlo (MC) simulations are widely used in polymer modeling [1] in order to extract the relevant thermodynamic and structural properties. The MC method is based on generating a random set of points in configuration space $x_{1}, x_{2}, \ldots, x_{n}$ (where $n$ is a large number) which resemble the distribution of such points in thermal equilibrium. The practical problem is to limit $n$, also referred to as the number of MC steps (MCS), to a manageable size, which would result in realistic times of simulation (we define a MC step as an attempt to move each particle in a simulated system). For example, the standard Metropolis Algorithm (MA) [1] provides a straightforward way to generate such points, but the number of MCS to reproduce the equilibrium distribution is often prohibitively large. While the MA works quite well for high temperatures (typically above a phase transition), it tends to generate traps in the local free energy minima for low temperatures (typically below a phase transition). Those traps often result in unreliable estimates of the sampled properties. To solve this problem, many modifica- tions of the MA were proposed [2-4]. One of those is parallel tempering (PT) method $[5,6]$, in which by parallel simulation of many replicas, in the relevant temperature range, the energy barriers can be overcome. In general, one simulates $M$ copies of a system for a set of temperatures, $T_{1}<T_{2}<\ldots<T_{M-1}<T_{M}$. The minimum and the maximum temperatures, $T_{1}$ and $T_{M}$, are fixed and the intermediate temperatures are selected to provide an optimal walk of replicas in the temperature space. The adjacent replicas are exchanged, at a given frequency in MCS, in random order with the following probability:

$$
p\left(T_{i}^{*} \leftrightarrow T_{i+1}^{*}\right)=\min \left[1, \exp \left(-\left(\beta_{i}-\beta_{i+1}\right)\left(U_{i+1}-U_{i}\right)\right)\right]
$$

where $\beta_{i}=1 / k_{B} T_{i}^{*}, k_{B}$ is the Boltzmann constant, and $U_{i}$ is potential energy of replica at $T_{i}^{*}$. If the system is trapped at lower temperature, it can be heated up in higher temperature and overcome the energy barrier. By using such scheme, one can get better statistics in less MCS, but one is required to simulate $M$ replicas in parallel. However, to run such simulation one has to set some parameters: number of replicas (temperatures) $M$, temperature set, $\left\{T_{1}, T_{2}, \ldots, T_{M}\right\}$, 
and exchange interval, $S$. It is known that quality of the PT method strongly depends on those parameters, and if they are not chosen correctly, simulation can give the same results as without the PT. It is not clear how many replicas are optimal, and it is hard to establish the appropriate criteria for this choice. One approach is to consider some thermodynamic quantity (for example the specific heat, $C_{v}$ ) and to test it how much CPU-time is needed to obtain correct result, or run many simulations with the same number of MCS (or total MCS of all replicas), and to test measurement accuracy for this quantity. In another approach, one can find the optimum $T^{*}$ set that gives the shortest round-trip times of replicas in temperature space.

Feedback-optimized PT (FOPT) algorithm, which we use in this work, is designed to find temperature sets that maximize the current of replicas from the lowest temperature to the highest one (details in reference [7]). In this algorithm we mark each replica with a flag up (when it reaches $T_{1}^{*}$ ) or down (when it reaches $T_{M}^{*}$ ). Initially the replicas do not have any flag, but they acquire one of them if any of the above conditions is met. During the simulation, we generate histograms $f_{u p}\left(T_{i}^{*}\right)$ and $f_{\text {down }}\left(T_{i}^{*}\right)$. Before exchanging replicas, we check all replicas and increment histograms in such way that $f_{u p}\left(T_{i}^{*}\right)$ is increased if replica with $T_{i}^{*}$ has an $u p$ flag, and $f_{\text {down }}\left(T_{i}^{*}\right)$ is increased if it has a down flag. If replica does not have any flag then neither of histograms is increased. From this data we create the $f\left(T_{i}^{*}\right)$ histogram:

$$
f\left(T_{i}^{*}\right)=\frac{f_{u p}\left(T_{i}^{*}\right)}{f_{\text {up }}\left(T_{i}^{*}\right)+f_{\text {down }}\left(T_{i}^{*}\right)} .
$$

The FOPT uses $f\left(T_{i}^{*}\right)$ histogram to optimize temperature set in order to minimize round-trip times.

In this work, we use round-trip time to find optimal parameters set. We test systems of different sizes, $N$, and with different number of replicas, $M$, to find the optimum parameter set. We do not vary the exchange interval, $S$, which is set to $200 \mathrm{MCS}$. In the future, we intend to test the $S$ effect on the PT simulation results.

It is worth to notice, that there are also other methods for generating optimal temperature sets [8-10]. One of them, is proposed by Sabe et al. [9], and is based mainly on the work of Kofke [11] and Kone and Kofke [12], in which the optimal temperature sets are found by using the condition of constant entropy increase for the adjacent replicas. According to our knowledge, the round-trip time of replicas with the FOPT, which we present in this work, is used as a criterion for finding optimal number of replicas in the PT simulations for the first time.

\section{MODEL}

The simulation is performed on the face centered cubic (FCC) lattice with coordination number $z=12$ and the bond length $l=a \sqrt{2}$ where $a$ is the FCC lattice constant. Chain bond are not allowed to be stretched or broken, and the usual periodic conditions are imposed. Lattice sites, which do not have the chain segments, are considered to contain the implicit solvent. The interaction is limited to the nearest neighbors $(z=12)$, and the interaction parameter, $\varepsilon$ is related to the Flory $\chi$ parameter by the following equation:

$$
\chi=\frac{(z-2) \varepsilon}{k_{B} T}
$$

This parameter, $\varepsilon$, serves also as an energy unit to define the reduced energy per segment, and the reduced temperature as

$$
\begin{aligned}
& \frac{E^{*}}{N}=\frac{(E / \varepsilon)}{N}, \\
& T^{*}=k_{B} T / \varepsilon,
\end{aligned}
$$

where, as before, $N$ is the number of chain segments.

In this simulation we use a single homopolymer chain with lengths $N=25,50$ and 75 segments on the FCC $30 \times 30 \times 30$ lattice. Elementary moves consist of pivot, kink and slithering-snake moves. Detailed description of this model can be found in references $[13,14]$

\section{RESULTS AND DISCUSSION}

\section{A. Simulation}

First, the system is equilibrated in athermal limit $\left(\varepsilon /\left(k_{B} T\right)=0\right)$, then we perform $2.5 \times 10^{5} \mathrm{MCS}$ to equilibrate system in the specified temperature range, and another $2.5 \times 10^{5} \mathrm{MCS}$ is used to sample data. During the simulation we collect data for $f\left(T_{i}^{*}\right)$ histogram and calculate the number of round trips that each replica performs. To get better $f\left(T_{i}^{*}\right)$ 's we assume that the minimum number of round-trips for each replica should be 4 . If this requirement is not met, we extend the simulation "time" by another $5 \times 10^{5}$ MCS. For each polymer length we perform many simulations with different number of replicas. For $N=25$ we use $M=6,8,10,12,14,16,20,24,28,32,40,50,60,70,80$ and 94 ; for $N=50$ we use $M=8,10,12,14,16,18,20,22$, $24,26,28,30,34,38,42,46,50,54,58,62,66,70,74,78$, 86 and 94 ; for $N=75$ we use $M=10,12,14,16,18,20,22$, 
$24,26,28,30,38,46,54,62,70,78,86$ and 94 . All systems are simulated in temperature range $T_{1}^{*}=1$ to $T_{M}^{*}=15$.

In each simulation we perform 6 rounds of the FOPT, starting with linear $T^{*}$ set chosen for the first round. We choose 6 rounds because, as seen in Figure 3, the temperature sets in round 4 and 5 are very similar, and we find that more rounds do not provide any improvement. In the last round we run $10^{7} \mathrm{MCS}$ to collect data to find optimal number of replicas. First $5 \times 10^{6} \mathrm{MCS}$ are used to equilibrate system and another $5 \times 10^{6} \mathrm{MCS}$ to collect the data (for longer chains and greater number of replicas we doubled or even tripled the number of MCS to obtain better statistics).

Before we show the results for optimal number of replicas, we look into the simulation process from the $1 \mathrm{st}$ round to the 5th round of the FOPT for chain length $N=75$ and $M=14$ and 30 (the second $M$ is used as a reference for simulation with $M=14$ ). In Figure 1 we present three traces of selected replicas in the $T^{*}$ space from the $1 \mathrm{st}$ round. As one can see each of the presented replicas reaches $T_{1}^{*}=1$ and $T_{M}^{*}=15$ many times, but more often it reaches higher temperatures.

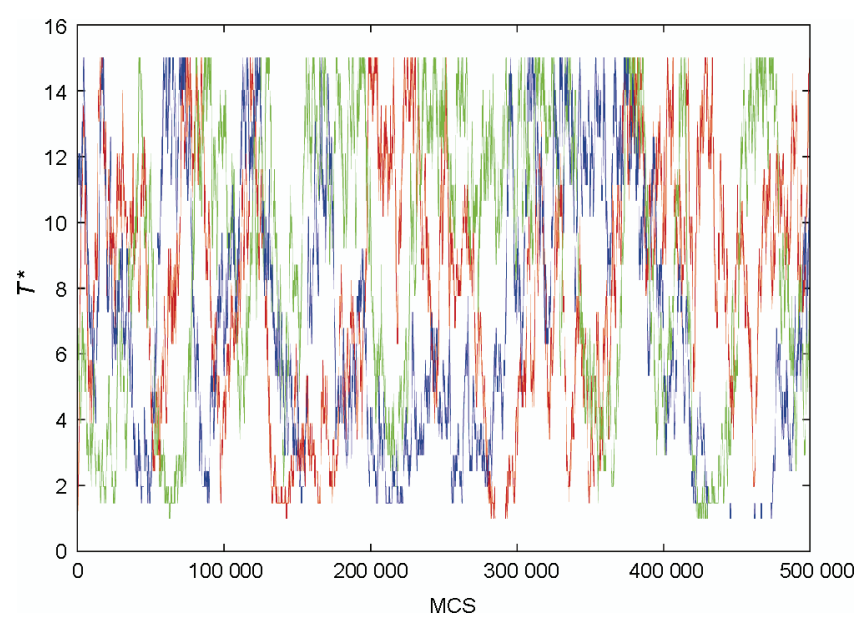

Fig. 1. Traces of three replicas in the temperature space for chain length, $N=75$, and the number of replicas, $M=30$, in the $1 \mathrm{st}$ round

To use the full potential of the PT method, the $T^{*}$ set should be optimized in such way that each replica spends about the same amount of time at each temperature. We use the FOPT algorithm for that goal. Figure 2 presents histogram $f\left(T_{i}^{*}\right)$, Figure 3 presents generated $T^{*}$ set for each simulation round and Figure 4 presents exchange probability between adjacent replicas in the 1st, the 2 nd and the 5 th round. In the 1 st round we use linear $T^{*}$ set, in next rounds temperatures are generated from the $f\left(T_{i}^{*}\right)$ histogram obtained in the previous round. The 2 nd round yields a reasonably optimized $T^{*}$ set, and in the next rounds, the $T^{*}$ set gets closer to the optimal solution. Black line in Figure 2 indicates ideal behavior. In the first round we can see that the $T_{1}^{*}$-replica is trapped and that its exchange probability with the $T_{2}^{*}$-replica is about $1 \%$. Exchange probabilities for other replicas are much higher and they grow with increasing $T^{*}$. In the 2 nd round the bottleneck at $T_{1}^{*}$ is removed and there is a significant improvement in the $f\left(T_{i}^{*}\right)$ histogram, but we can notice another bottleneck at $T_{9}^{*}$ with the exchange probability of about $9 \%$. This bottleneck is removed in the following rounds. As seen from

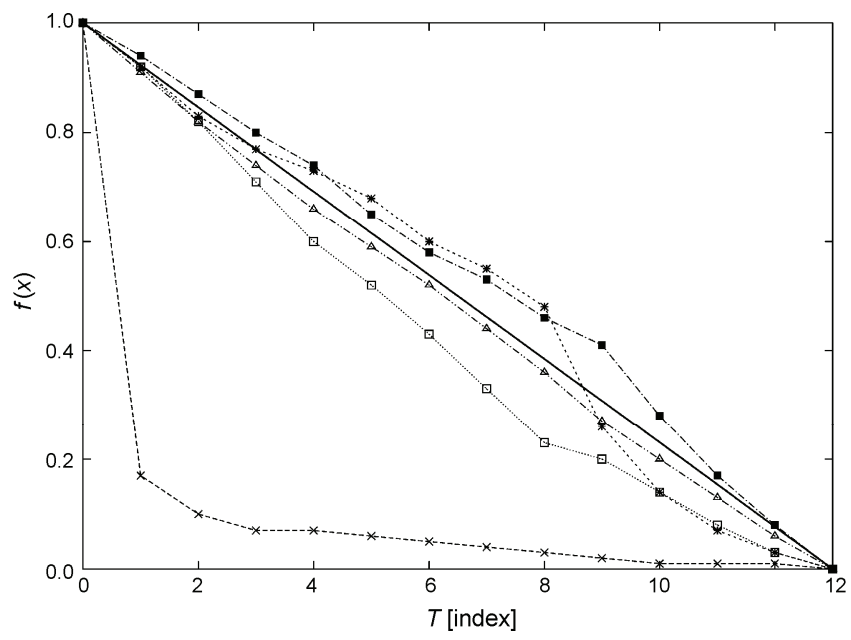

Fig. 2. The $f\left(T_{i}^{*}\right)$ histogram for each round of the FOPT simulation for $N=75$ and $M=14$ : the 1st round (dashed line with crosses), the 2nd round (short dashed line with stars), the 3rd round (dotted line with squares), the 4 th round (dash-dotted line with filled squares), the 5th round (dash-double-dotted line with triangles) and a reference (solid line)

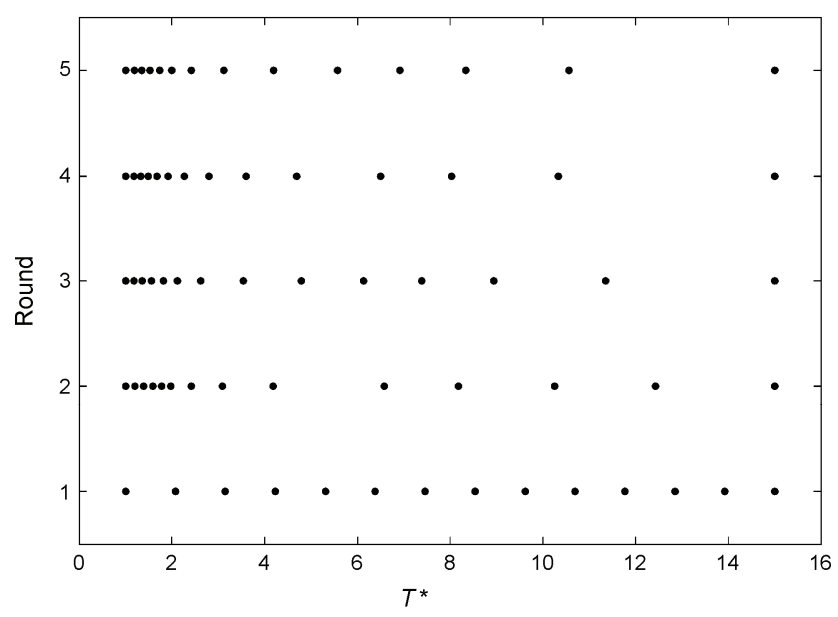

Fig. 3. The temperature set for each round of the FOPT simulation for $N=75$ and $M=14$. In the first round we start with linear temperatures, and in next rounds temperatures are generated with the FOPT algorithm 


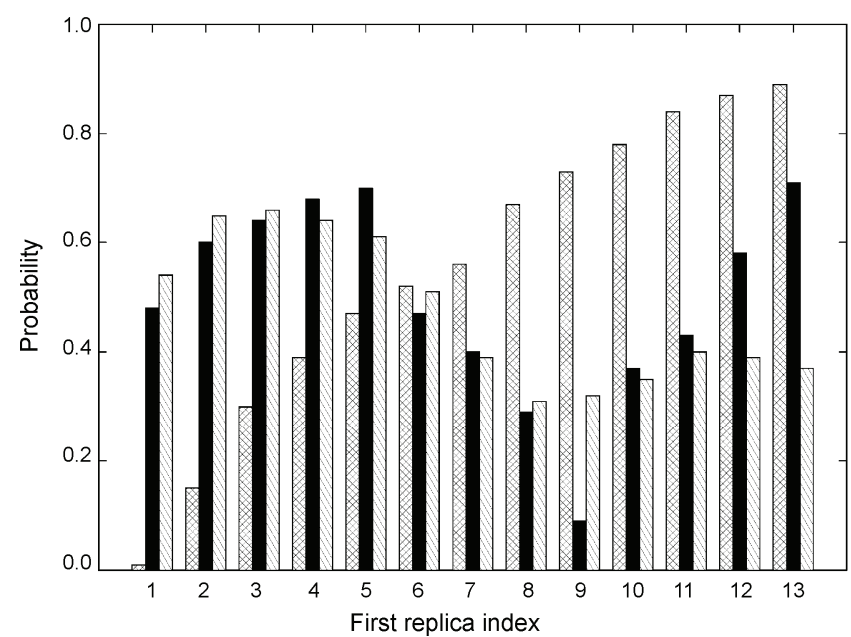

Fig. 4. Exchange probability for three FOPT rounds for $N=75$ and $M=14$. Boxes filled with crossed lines are for the 1 st round, boxes filled with black are for the 2 nd round and boxes filled with lines are for the 5 th round

exchange probability for the 5th round, the optimal solution has varying exchange probabilities. In general, they should be higher for lower temperatures and for $T_{i}^{*}$ with larger specific heat.

\section{B. The long chain properties}

Now we focus on selected thermodynamic and structural properties of the polymer chain of length $N=75$ with $M=14$ replicas. It is well known that a single homopolymer chain undergoes coil-to-globule transition upon cooling (in general, upon decreasing the solvent

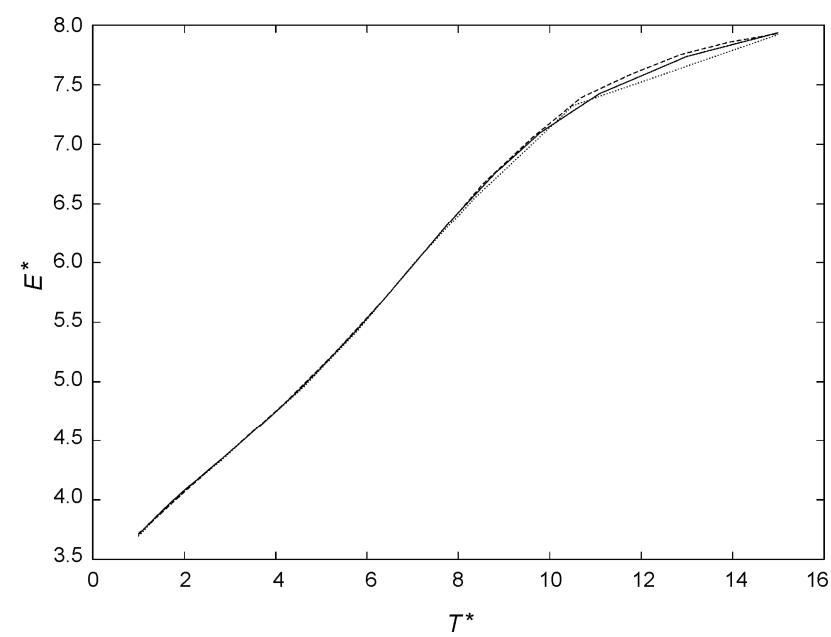

Fig. 5. Reduced energy per segment $E^{*} / N$ as a function of temperature $T^{*}$ for chain length $N=75$ with number of replicas $M=14:$ the 1st FOPT round (dashed line), the 5th round (dotted line) and a reference (solid line). For the reference we use $M=30$ and $2 \times 10^{7} \mathrm{MCS}$

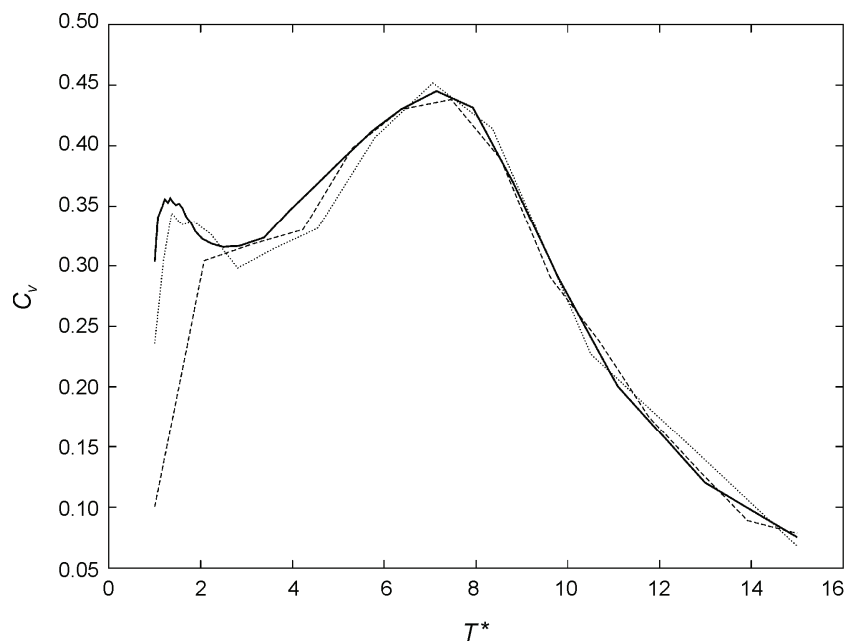

Fig. 6. Specific heat, $C_{v}$, as a function of temperature, $T^{*}$, for chain length, $N=75$, with the number of replicas, $M=14$ : the 1st FOPT round (dashed line), the 5th round (dotted line) and a reference (solid line). For the reference we use $M=30$ and $2 \times 10^{7} \mathrm{MCS}$

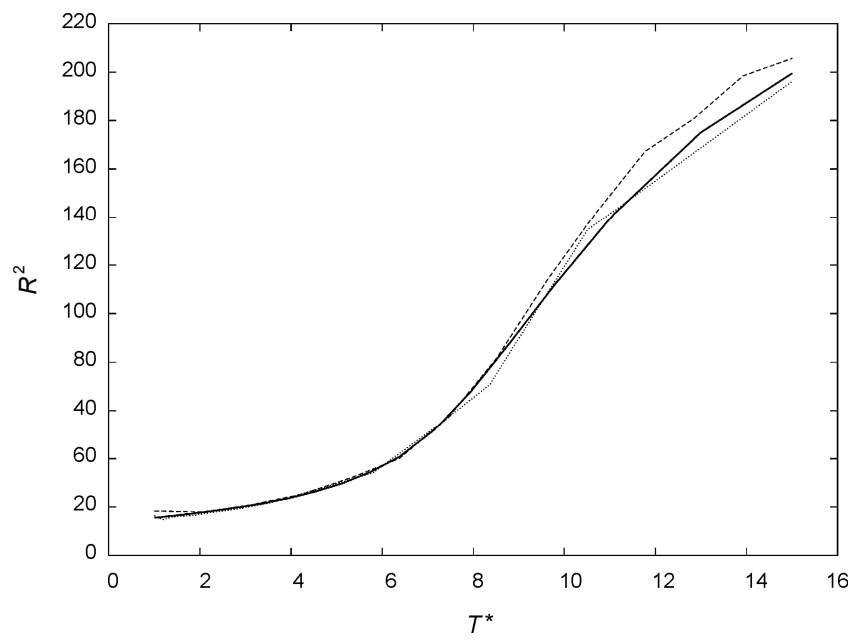

Fig. 7. Squared end-to-end distance as a function of temperature, $T^{*}$, for chain length $N=75$ with number of replicas $M=14$ : the 1st FOPT round (dashed line), the 5th round (dotted line) and a reference (solid line). For the reference we use $M=30$ and $2 \times 10^{7} \mathrm{MCS}$

quality). In Figure 5 we present reduced energy per segment, in Figure 6 the specific heat, and in Figure 7 the squared end-to-end distance for 1 st round (dashed), for the 5th round (dotted), and for the 6th round after $2 \times 10^{7} \mathrm{MCS}$ (solid) that is treated as a reference. From $T^{*}=15$ to 7.2 the chain is in the swollen coiled state, then at about $T^{*} \approx 7.2$ it undergoes coil-to-globule transition. For lower $T^{*}$ it is in a globular state, but at about $T^{*} \approx 1.4$ there is another ordering effect, probably crystallization on the FCC latice. As seen in Figure 8, globule below this temperature is highly ordered. As seen in Figure 5, the 
energy for each round is almost the same, but the specific heat is not. Since in the 1 st round we use linear $T^{*}$ set, we miss the low temperature ordering effect at about $T^{*} \approx 1.4$. Because the $T_{1}^{*}$-replica is most likely trapped in a free energy minimum, probing specific heat for $T_{1}^{*}$ gives a wrong result. But with optimized $T^{*}$ set for the 5 th round, we observe a considerable improvement of the specific heat for the same number of MCS.
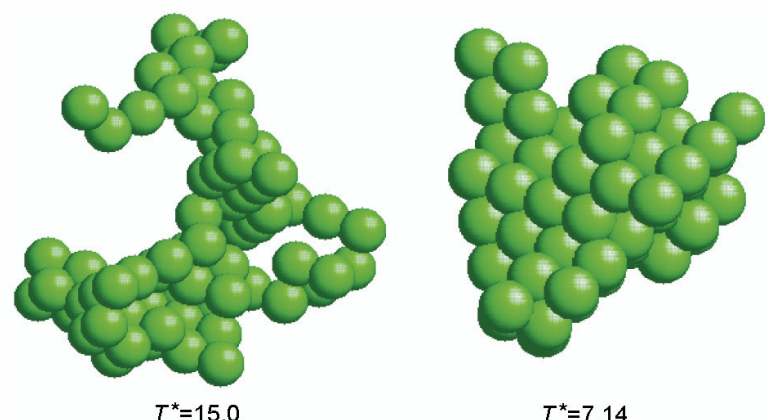

$T^{*}=7.14$

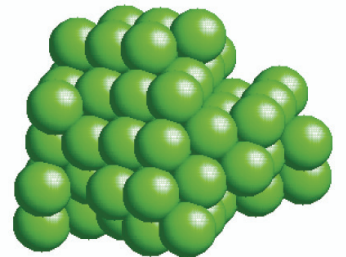

$T^{*}=2.49$

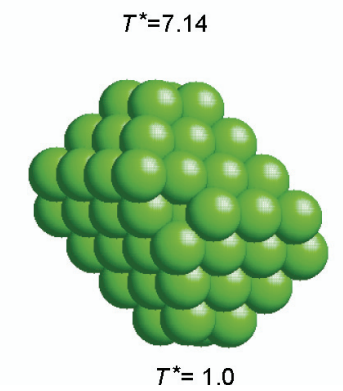

Fig. 8. Snapshots of structures of polymer chain of length $N=75$ for temperatures: $T^{*}=15$ (swollen state), $T^{*}=7.14$ (coil-toglobule transition), $T^{*}=2.49$ (globular state) and $T^{*}=1.0$ (ordered globular state below $T^{*} \approx 1.4$ )

As we can see, the PT method without optimization also gives reasonable results. It would be, therefore, reasonable to compare simulation results from simulation with and without the PT. Such comparisons can be found, for example, in reference [15]. It is worth to notice that the FOPT not only speeds up the simulation process (giving better sampled data approximation) but also it sets temperatures in such way that the relevant temperature ranges are sampled more accurately (by arranging temperatures in areas where the specific heat is higher).

\section{Optimal number of replicas}

To find optimal number of replicas for each system size, we perform simulations with different number of replicas and in each case we measure the round-trip time (to get from $T_{1}^{*}$ to $T_{M}^{*}$, and then back from $T_{M}^{*}$ to $T_{1}^{*}$ ). Figure 9 presents average round-trip time for polymers length $N=25$ (solid line), 50 (dashed line) and 75 (dotted line). For each $N$, the smallest possible number of replicas is chosen to run the PT so that the round-trip is actually performed. For smaller $M$ 's, the replicas cannot perform any round-trips in the temperature space. However if we had a better $T^{*}$ set in the 1st round, it might run correctly. As we can see in Figure 9 when we increase the chain length, the replicas need more MCS to perform one roundtrip. For $N=25,50$ and 75 the smallest round-trip time is $21 \times 10^{3}, 58 \times 10^{3}$ and $136 \times 10^{3} \mathrm{MCS}$, respectively. Thus it seems to increase in a non-linear way. Also from Figure 9, we can see that with increasing number of replicas, the round-trip time is also increased. This is because a single replica has to take a longer path as $M$ is increased. For example, for $M=10$ it is only 9 steps in one direction, and another 9 in the other to complete a single round-trip, whereas for $M=50$ it is 98 steps in both directions. From this plot it is not clear what is the optimal $M$. Therefore we report the round-trip time divided by $2(M-1)$ (Figure 10) in order to get time needed to perform a single step in $T^{*}$ space. Figure 10 shows a clear minimum of the MCS time at a given $M$, for each $N$. While for $N=25$ and $M=6$, it takes about 2100 MCS to perform a single step in $T^{*}$ space, when we increase $M$ we reach a minimum for $M=12$ with $1280 \mathrm{MCS}$. For higher $M$ we observe an increase of time needed to perform a single step. For $N=50$ the minimum number of replicas is $M=8$, and in this case it takes $4150 \mathrm{MCS}$ per single step. Again, with increasing $M$, the number of MCS decreases, and for $M=20$ it reaches a minimum with $2370 \mathrm{MCS}$. For $N=75$, we observe a similar behavior with minimum for $M=30$ at 3930 MCS. By dividing the minimum MCS by chain length, $N$, we obtain a value about 50 (Fig. 11).

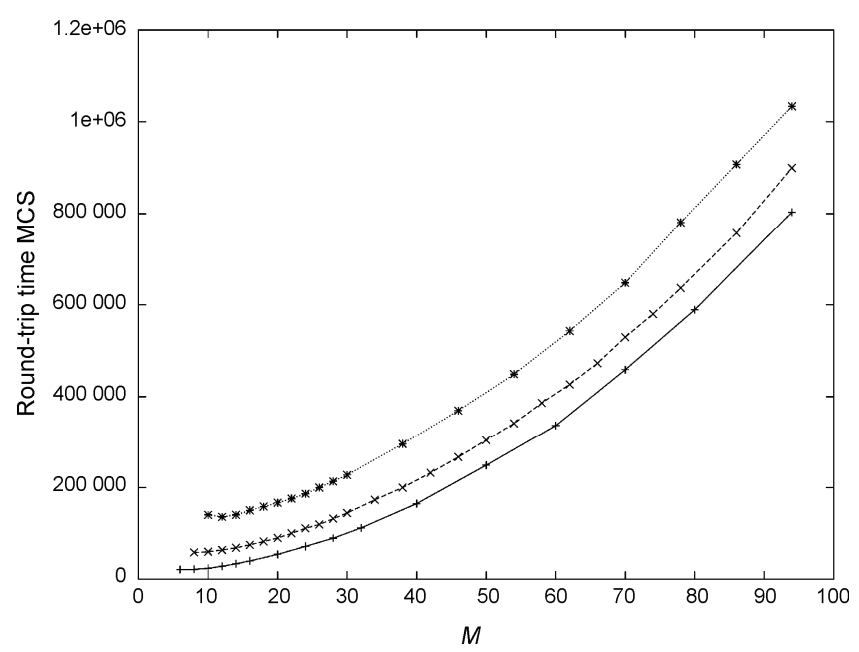

Fig. 9. Round-trip time in MCS as a function of number of replicas $M$ for chain lengths: $N=25$ (solid line), $N=50$ (dashed line) and $N=75$ (dotted line) 


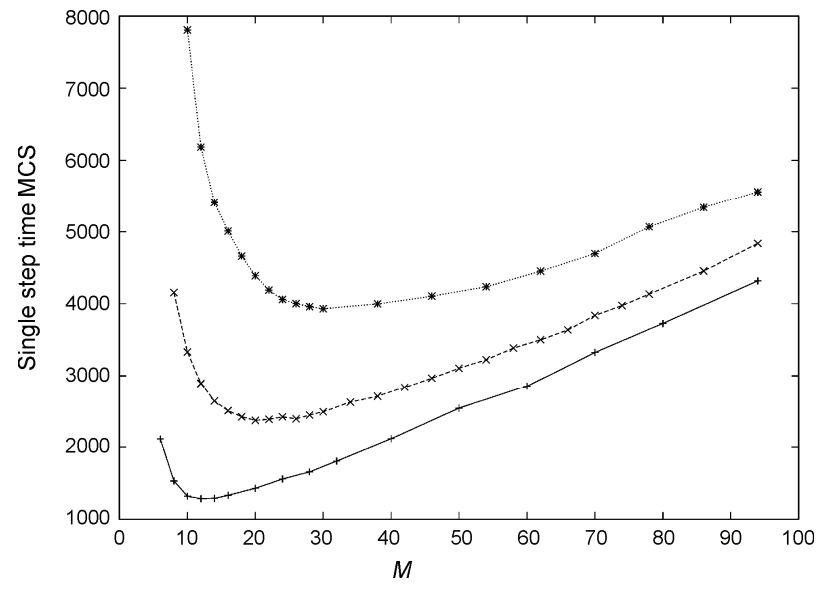

Fig. 10. Average time in MCS needed to perform a single step in temperature space as a function of number of replicas, $M$, for chain lengths: $N=25$ (solid line), $N=50$ (dashed line) and $N=75$ (dotted line)

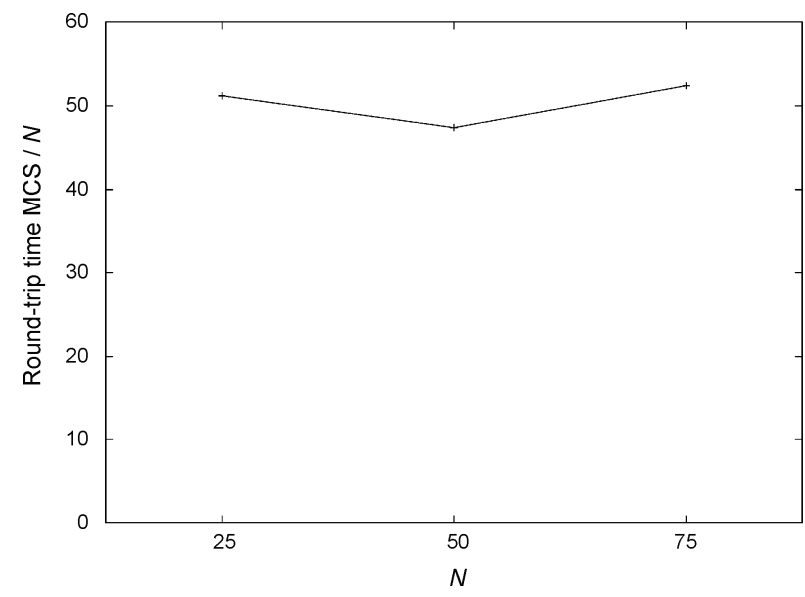

Fig. 11. Minimum average time needed to perform a single step in temperature space divided by chain length $N$ as a function of $N$

It is known that number of replicas grows like $\sqrt{N}$ where $N$ is a size of a system $[6,11]$. In principle, if one can find the optimal number of replicas for specified $N$, and then one can estimate the optimal $M$ for any $N$. However, in our simulations it is not confirmed. If we take $N=25$ with optimal $M=12$, then optimal $M$ for $N=50$ and 75 should be 17 and 21, respectively. However, it is known that with increasing polymer chain length, coil-to-globule transition moves to higher temperatures, so it has different specific heat which affects the optimal $M$. Another explanation could be that our choice for definition of optimal $M$ is not the same as in the other studies [16]. On the other hand, we have only 3 chain lengths so it is quite difficult to verify this scaling with only 3 points. In our simulations we get an approximate "scaling":

$$
M \sim N
$$

Average exchange probability between adjacent replicas for chain lengths $N=25,50$ and 75 is $64 \%, 70 \%$ and $74 \%$, respectively. This is quite large compared to results obtained by Kone and Kofke [12] and by Rathore et al. [16] for systems with constant specific heat. They found that optimal exchange probability is about $23 \%$.

\section{CONCLUSIONS}

We perform Monte Carlo simulations of homopolymer chains of lengths $N=25,50$ and 75 in temperatures range $T_{1}^{*}=1$ to $T_{M}^{*}=15$ and find the optimal number of replicas, $M$, based on the shortest time needed to perform a single step in the temperature space. We also find that for each chain length, $N=25,50$ and 75 , the optimal $M$ is 12,20 and 30, respectively. The optimal $M$ is found to be approximately proportional to $N, M \sim N$ relation.

However, if one changes temperature range, then a set of the FOPT simulations should be run again to find optimal $M$ for this system. For example, in this work we obtain that the optimal $M=12$ for $N=25$, but if we set $T_{1}^{*}=0.5$ and $T_{M}^{*}=10$, we would get different optimal $M$. Finding the optimal $M$ for different $T^{*}$ ranges is not a subject of this work, but it may be crucial. Another significant parameter which is not changed during the simulation is the replica exchange interval, $S$.

The FOPT is a promising method for simulating polymer systems. This algorithm provides an iterative way to find optimal number of replicas, but it is quite CPU-time consuming (one has to perform numerous simulations to prepare an optimized $T^{*}$ set in which the targeted system is sampled). It can also be unstable, and sometimes the generated temperatures can be so inappropriate that the free energy bottlenecks are formed, which are difficult to overcome by the replicas' walk in the $T^{*}$ space.

\section{Acknowledgments}

We gratefully acknowledge the computational grant from the Poznan Supercomputing and Networking Center (PCSS) and grant N202 287338 from Polish Ministry of Science and Higher Education.

\section{References}

[1] K. Binder, W. Paul, Macromolecules 41, 4537 (2008).

[2] F. Wang, D.P. Landau, Phys. Rev. Lett. 86, 2050 (2001).

[3] V. Cerny, Journal of Optimization Theory and Applications 45, 41 (1985). 
[4] S. Kirkpatrick, C.D. Gelatt Jr., M.P. Vecchi, Science 220, 671 (1983).

[5] R.H. Swendsen, J.S. Wang, Phys. Rev. Let. 57, 2607 (1986).

[6] D.J. Earl, M.W. Deem, Phys. Chem. Chem. Phys. 7, 3910 (2005).

[7] H.G. Katzgraber, S. Trebst, D.A. Huse, M. Troyer, J. Stat. Mech. p. P03018 (2006).

[8] D. Gront, A. Kolinski, J. Phys. Condens. Matter 19 (2007).

[9] D. Sabo, M. Meuwly, D.L. Freeman, J.D. Doll, J. Chem. Phys. 128 (2008).

[10] C. Predescu, M. Predescu, C.V. Ciobanu, J. Phys. Chem. B 109, 4189 (2005).
[11] D.A. Kofke, J. Chem. Phys. 117, 6911 (2002), 120, 206101 (2004).

[12] A. Kone, D.A. Kofke, J. Chem. Phys. 122, 1 (2005).

[13] S. Wołoszczuk, M. Banaszak, P. Knychała, K. Lewandowski, M. Radosz, J. Non-Cryst. Solids 354, 4138 (2008).

[14] K. Lewandowski, P. Knychała, M. Banaszak, Phys. Stat. Sol. B 245, 2524 (2008).

[15] A. Sikorski, Macromolecules 35, 7132 (2002).

[16] N. Rathore, M. Chopra, J.J. de Pablo, J. Chem. Phys. 122, 024111 (2005).
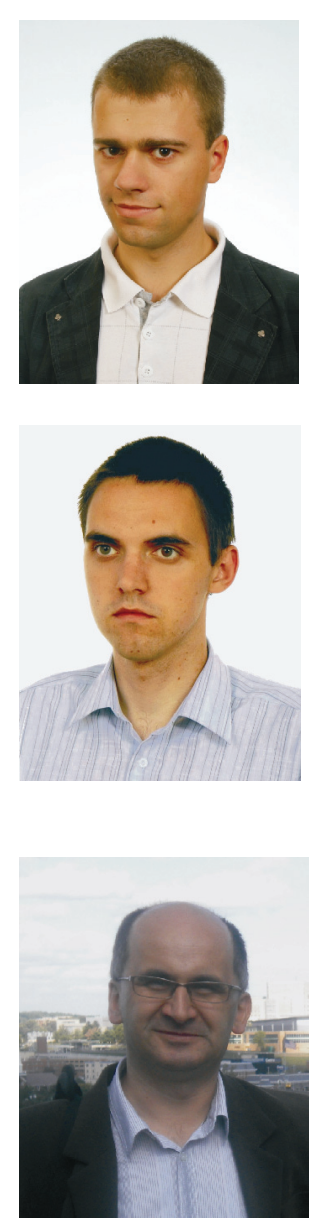

KrZYSZTOF LewANDOwSKI, received his MSc degree in Computer Science in 2007 from the Adam Mickiewicz University in Poznań where he is a Ph. D. student in Physics. His research interests concern polymer physics, globular and micellar nanostructures, parallel algorithms and programming.

Piotr KnychaŁA, received his MSc degree in Computer Science in 2007 from the Adam Mickiewicz University in Poznań where he is a Ph. D. student in Physics. The main research interests include polymer melts and solutions, block copolymer nanostructures, ionic copolymers, coarse-grained models of biopolymers investigated by the lattice computer simulations.

Michal Banaszak, graduated in Theoretical Physics in 1985 from Adam Mickiewicz University in Poznań. He received his PhD degree in Physics in 1991 from Memorial University in St. John's, Canada, specializing in Polymer Physics. From 1992 to 1995 he worked as postdoctoral fellow in Exxon Research \& Engineering Co. in Annandale, New Jersey, USA, focusing on theory and modeling of polymer solutions. From 1995 to 1997 he worked in the Chemistry Department of UMIST in Manchester, UK, as a research associate, specializing in large-scale computer simulations of ionic copolymers. In 1997 he joined the Adam Mickiewicz University, obtaining DSc degree (habilitation) in 2004 in Physics (specialty: Soft Matter Physics and Computer Simulations). Currently he is involved in a variety of research projects on polymer modeling, collaborating with research groups from leading universities, such as University of California in Berkeley. His main interest is in developing new models and theories for nanoscale self-assembly of various copolymer systems. He also successfully interacts with experimentalists, using large-scale computing and developing novel efficient algorithms and codes. 